\title{
Adaptation de conduites d'élevage ovin allaitant à une diminution du chargement. Exemple en moyenne montagne
}

\author{
Alain Brelurut ${ }^{\mathrm{a} *}$, Frédérique Louault ${ }^{\mathrm{b}}$, Marc Benoit ${ }^{\mathrm{c}}$, \\ Hervé Tournadre ${ }^{\mathrm{a}}$, François Xavier de Montard ${ }^{\mathrm{b}}$, Michel Thériez ${ }^{\mathrm{a}}$, \\ Gilbert Liénard $^{c}$, Benoît Dedieu $^{\mathrm{a}}$, Gabriel Laignel ${ }^{\mathrm{c}}$
}

\author{
${ }^{a}$ Adaptation des herbivores aux milieux, Inra, Theix, 63122 Saint-Gènes-Champanelle, France \\ b Agronomie, Inra Clermont-Ferrand-Theix, France \\ ' Économie de l'élevage, Inra Clermont-Ferrand-Theix, France
}

(Reçu le 11 mai 1998 ; accepté le 27 juillet 1998)

\begin{abstract}
Adjustment of suckler sheep management to a decrease of stocking rate: example in middle mountain. The results of two long-term studies investigating the effect of suckling ewe stocking rate on show that when the stocking rate of pastures is significantly decreased $(1.2 ; 0.85$; and $0.6 \mathrm{LSU} \cdot \mathrm{ha}^{-1}$ ) by increasing the surface area, the input of nitrogen fertilizer and concentrate feedstuffs can be reduced. Successful herd management maintained a high breeding rate (1.6 lambs/ewe/year), while preserving the pasture land over the whole area. However, at $0.6 \mathrm{LSU} \cdot \mathrm{ha}^{-1}$ the difference between the available food and the needs of the flock results in the pasture being consumed at a lower rate ( $37 \%$ compared with $61 \%$ at 1.2 and $0.85 \mathrm{LSU} \cdot \mathrm{ha}^{-1}$ ). Under these conditions, the risk of rapid deterioration of the pasture land is high. Changes to the reproduction calendar produced an increase in the amount of pasture consumed (farming exclusively outside and winter grazing), which reduced but not eliminated the risk of rapid pasture deterioration. The results indicate that on pastures where grazing pressure is lowest, there will be a reduction in the quality of the pasture and an increase in scrub. (C) Elsevier / Inra
\end{abstract}

suckler sheep / stocking rate / herd management / middle mountains

Résumé - Les résultats de deux essais de longue durée sur brebis allaitantes montrent qu'une diminution importante du chargement $\left(1,2 ; 0,85\right.$ et $\left.0,6 \mathrm{UGB} \cdot \mathrm{ha}^{-1}\right)$, obtenue par un agrandissement, constitue une opportunité de forte réduction des intrants, fertilisants azotés et aliments concentrés. Les conduites de troupeaux mises en ceuvre ont permis de maintenir les performances zootechniques à un niveau élevé (1,6 agneaux nés par brebis et par an), tout en favorisant le pâturage de façon à entretenir

* Correspondance et tirés à part

Tél. : (33) 0473624061 ; fax : (33) 0473624118 ; e-mail : brelurut@ clermont.inra.fr 
la totalité du territoire, et en améliorant l'autonomie fourragère. Toutefois, à 0,6 UGB $\cdot$ ha $^{-1}$, l'importance de l'écart entre la production fourragère et les besoins se traduit par une forte diminution du taux d'utilisation de la ressource ( $37 \%$ au lieu de $61 \%$ à 1,2 et à 0,85 UGB $^{-1} a^{-1}$ ). Dans ces conditions, le risque de dégradation rapide des prairies devient très important. De profondes modifications du calendrier de reproduction permettant d'accroître le prélèvement par le pâturage (plein air intégral et pâturage d'hiver), ont permis de diminuer ce risque sans l'écarter totalement. Les évolutions, à confirmer à terme, indiquent en deux ans une baisse de la qualité des prairies et une extension des ligneux sur les zones où la pression de pâturage est la plus faible. (C) Elsevier / Inra

\section{élevage ovin / chargement / conduites d'élevage / moyenne montagne}

\section{INTRODUCTION}

Face aux perspectives d'agrandissement des exploitations des zones herbagères, deux essais de longue durée en élevage ovin ont été menés à l'Inra, pour raisonner les adaptations des conduites permettant l'intégration de nouvelles surfaces et en mesurer les effets sur les performances économiques et techniques.

La baisse du chargement, obtenue par agrandissement des surfaces à troupeau constant, permet d'accroître l'offre fourragère de l'exploitation, mais la nature des questions posées par la modification du rapport entre production et besoin des troupeaux est différente selon le chargement initial.

Les niveaux retenus dans les essais, de 0,85 (E1) et de 0,6 UGB $\cdot$ ha $^{-1}$ (E2), sont nettement plus bas que celui du témoin (T) géré à $1,2 \mathrm{UGB} \cdot h^{-1}$. Ils correspondent à deux phases successives d'agrandissement brutal des surfaces et permettent de préciser les enjeux et des voies d'adaptation propres de deux cas d'agrandissement.

- Dans le premier, le chargement initial (T), comparable à la situation observée dans des réseaux d'exploitation de moyenne montagne [2], correspond à un élevage conduit avec un chargement relativement élevé dans le milieu considéré, avec utilisation d'intrants (fertilisation, aliments concentrés) pour atteindre les objectifs zootechniques. L' agrandissement des surfaces fourragères peut alors être mis à profit pour réduire ces charges. C'est la situation créée dans le premier essai avec les dispositifs $\mathrm{T}$ et El $[7,10]$. Pour El, l'enjeu est de préciser les conditions d'intégration des surfaces supplémentaires jusqu'à atteindre un nouvel équilibre permettant, à moindre coût, de maintenir le niveau des performances zootechniques et de conserver la qualité de la ressource fourragère pour la production d'agneaux.

- Le second correspond à un agrandissement au niveau d'une exploitation déjà peu chargée et utilisant peu d'intrant. Le dispositif E2, à 0,6 UGB·ha ${ }^{-1}$, se place à un niveau faible de chargement ; l'excès d'offre fourragère risque alors d'entraîner une baisse globale de la qualité des prairies et le développement de l'enfrichement. L'objectif d'entretien de la totalité de la surface devient plus difficile à atteindre que dans le cas précédent, et risque d'imposer aux animaux des contraintes fortes, pouvant être à l'origine de la dégradation de leurs performances. Ces contraintes sont d'autant plus fortes qu'une partie des surfaces additionnelles est géographiquement éloignée du noyau de l'exploitation, comme on le rencontre souvent dans la pratique. Dans ce cas, l'enjeu est donc d'identifier et de mettre en œuvre des conduites, qui permettraient, essentiellement par l'action des troupeaux, de préserver la qualité de la ressource sans dégrader les performances zootechniques.

Cet article a pour objectifs de décrire les adaptations mises en œuvre pour répondre à ces situations d'agrandissement, et d'exposer les conséquences de ces modes de gestion sur les performances animales et sur le milieu. 


\section{CONDITIONS EXPÉRIMENTALES}

Le premier essai s'est déroulé de 1988 à 1992 et le second, qui a débuté en 1994 et doit se poursuivre jusqu'en 1999, sont conduits sur un domaine expérimental de l'Inra, situé dans le Massif Central, à $750 \mathrm{~m}$ d'altitude, sur terrains granitiques; la pluviométrie et la température moyennes annuelles sont de $760 \mathrm{~mm}$ et de $8,6^{\circ} \mathrm{C}$.

Le territoire est hétérogène, avec des secteurs précoces et frais en été, et d'autres plus tardifs sur sols superficiels. Les surfaces mécanisables représentent $65 \%$ de la surface totale en $\mathrm{T}$ et $\mathrm{E} 1$ et $50 \%$ en $\mathrm{E} 2$, le reste ne pouvant être que pâturé. Sur E2, $30 \%$ de la surface totale sont éloignés du site principal d'exploitation. Les ligneux bas sont présents à l'état de début d'enfrichement, sur la plupart des pacages et parcours, avec un recouvrement initial d'environ $5 \%$ de la surface des parcelles.

Les brebis de race rustique Limousine et de format moyen $(60,8 \mathrm{~kg}$ en moyenne) présentent une bonne aptitude maternelle et un potentiel élevé de productivité numérique permettant de mettre en évidence d'éventuels effets de la conduite sur les performances. Le rythme de reproduction est d'un agnelage par an, avec deux périodes de mises bas (printemps et automne). Ce choix résulte d'une volonté de répondre aux besoins du marché avec une production relativement étalée, et de disposer en permanence de lots d'animaux aux besoins contrastés apportant une grande souplesse d'utilisation des ressources fourragères de qualités différentes.

L'alimentation repose sur une utilisation maximale des fourrages, avec le souci d'une production de qualité pour les catégories d'animaux à haut niveau de besoins (brebis en fïn de gestation et en début d'allaitement, agneaux sevrés). Les agneaux d'automne qui sont élevés en bergerie disposent d'une ration à base d'aliments concentrés.

Les abattages d'agneaux sont réalisés au poids et à l'état d'engraissement corres- pondant au mieux à la demande du marché national, et dans une moindre mesure à celle du marché espagnol (agneaux de $25 \mathrm{~kg}$ environ).

La totalité de la surface fourragère est exploitée au moins une fois par an. Le pâturage des surfaces éloignées de E2 est réalisé, pour des raisons de commodité et d'organisation du travail, avec des animaux peu exigeants en surveillance (brebis vides ou en début de gestation ou en cours de lactation) entre avril et octobre. Le pâturage tournant est imposé par le parcellaire et l'hétérogénéité du territoire.

\section{ADAPTATIONS DES CONDUITES}

Les adaptations ont été raisonnées avec le souci d'assurer la pérennisation des systèmes extensifiés à travers le renouvellement de ressources de qualité et de maintenir le niveau de productivité animale dont dépend fortement le revenu de l'éleveur en système allaitant ovin [1]. C'est pourquoi ont été visés tout particulièrement le maintien de performances zootechniques d'un bon niveau par rapport au potentiel génétique de la race utilisée, la valorisation maximum de la ressource fourragère et l'utilisation de la totalité du territoire disponible pour en assurer l'entretien.

Les troupeaux constituent l'outil privilégié de valorisation et d'entretien des surfaces. Les adaptations principales à caractère zootechnique visent à augmenter les prélèvements directs au pâturage en allongeant les périodes de séjour des animaux sur les parcelles. Elles portent done surtout sur le choix des dates de reproduction, qui conditionnent les niveaux et la répartition dans le temps des besoins alimentaires.

En El, la plus grande disponibilité de surface permet d'organiser une mise à l'herbe plus précoce qu'en T, les faibles hauteurs d'herbe offertes étant compensées par des surfaces visitées plus importantes. La date des mises bas de printemps de El a 
donc été avancée de deux semaines, afin de disposer relativement tôt de brebis à forte capacité d'ingestion et de déprimer les parcelles à pousse précoce (tableau I). On cherche également à réaliser la finition des agneaux de printemps à l'herbe, sur des parcelles de repousses préparées à cette fin. Toutefois, si la qualité ou la quantité de fourrages sur pied est insuffisante, la finition est assurée par un apport complémentaire d'aliments concentrés à l'herbe, ou par l'entrée en bergerie.

Pour E2, l'écart important, surtout au printemps, entre la production fourragère et la capacité de prélèvement du troupeau, l'hétérogénéité du territoire, et aussi l'éloignement de certaines parcelles, imposent des contraintes fortes au niveau du calendrier de pâturage. Afin de répondre aux objectifs d'entretien de la totalité de la surface, l'exploitation des parcelles a été organisée en définissant des priorités. Les pacages et parcours sont exploités en priorité, les parcelles fauchables pouvant être nettoyées par la fauche. Parmi ces pacages, ceux exposés à l'envahissement des ligneux sont visités régulièrement dès le début de la saison de pâturage afin que ces arbustes, porteurs des jeunes pousses, restent attractifs pour les animaux. Les surfaces éloignées font l'objet d'un pâturage prolongé en fin de printemps et en été, aux dépens des parcelles proches où l'exploitation partielle de début d'année est suivie de pâturages d'automne et d'hiver, permettant de limiter l'accumulation prolongée de ressources sur pied.

La nécessité de disposer de brebis à faibles besoins, en hiver et début de printemps, a donc entraîné le report de l'agnelage de printemps au mois de mai, avec mise bas en extérieur à l'herbe. La lutte de décembre se pratique en monte libre, sans synchronisation des chaleurs afin d'étaler les naissances pour réduire les risques de mortalité des agneaux due aux intempéries. Ce troupeau est donc conduit en plein air intégral, contrairement à celui qui met bas en automne, et qui entre en bergerie pour l'agnelage et la lactation. Les brebis concernées restent au pâturage jusqu'au début du mois d'octobre, puis, après le tarissement en janvier, rejoignent le troupeau de plein air. Les durées de stabulation pour E2 sont donc fortement réduites (tableau I).

Les fauches sont en premier lieu réalisées pour constituer les stocks. Pour El et surtout pour E2, on recherche des rendements faibles, de façon à gérer par la fauche des surfaces importantes, grâce à des coupes de repousses après déprimage sur des parcelles peu fertilisées. Les fauches précoces d'excédents peuvent aussi être effectuées pour préserver la qualité des ressources à pâturer ; en E1, elles peuvent être laissées sur le terrain.

Compte tenu des disponibilités fourragères, la fertilisation azotée a été réduite dans le cas des faibles chargements, passant (en moyenne annuelle) de $67 \mathrm{~kg} \mathrm{~d} N \cdot \mathrm{ha}^{-1}$ pour $\mathrm{T}$, à 18 en E1 et 0 en E2. Cela permet de limiter les masses d'herbe à gérer, et participe à la réduction des intrants. Parallèlement, le développement du trèfle blanc est encouragé dans les systèmes moins chargés afin de conserver à terme la qualité des fourrages et la fertilité du sol. Pour cela, les fertilisations en $\mathrm{P}$ et $\mathrm{K}$ ont été maintenues, et un sursemis à la volée a été effectué sur quelques parcelles en E1. Les apports d'azote dans E1, ciblés sur certaines parcelles, sont maintenus pour favoriser la mise à l'herbe précoce des brebis allaitantes, pour produire des repousses pour la finition des agneaux de printemps, et, si nécessaire, pour accroître le rendement des récoltes.

\section{RÉSULTATS}

\subsection{Performances animales}

Globalement, les performances zootechniques se maintiennent, quelque soit le niveau de chargement. La fécondité des brebis se situe à un niveau élevé et comparable entre T, E1 et E2 (tableau I). Seule la mor- 
talité de E2 est plus importante, surtout du fait des pertes par des prédateurs sauvages lors des mises bas de mai.

Les agneaux de E1, conduits à l'herbe ou avec des rations essentiellement constituées de fourrages, présentent des performances de croissance élevées et comparables à celles de $\mathrm{T}$, au moins jusqu'à l'âge de 70 jours. Au-delà, leurs performances sont moindres, ce qui retarde l'âge à l'abattage, mais permet de produire des animaux légèrement plus lourds (tableau I). La croissance des agneaux de E2 est également d'un niveau comparable.

Les agnelles de reproduction, conduites avec les agneaux de boucherie, présentent pour la plupart un développement corporel insuffisant pour une première saillie à l'âge initialement fixé de huit mois. Plutôt que de compenser ce retard par un apport massif d'aliments concentrés, il a été décidé de ne présenter à la lutte à huit mois que les agnelles les plus lourdes, et d'accorder six mois supplémentaires aux plus légères. Cette modification de la conduite entraîne une très forte diminution de productivité des agnelles de E1 et E2 à l'âge de 13 mois (1,24 agneau produit par agnelle de $\mathrm{T}$, contre seulement 0,15 pour E1 et E2), ensuite compensée à partir de 30 mois par une fécondité plus élevée. De même, les écarts de taille au garrot observés dans le jeune âge disparaissent totalement à l'âge adulte.

Les modes de conduite mis en œuvre permettent de réduire fortement les apports d'aliments concentrés pour E1 et E2, avec seulement 79 et $59 \mathrm{~kg}$ par brebis, contre $108 \mathrm{~kg}$ pour T (tableau I), soit une diminution de 27 et de $45 \%$. L'écart provient de la moindre consommation d'aliments des brebis qui mettent bas au printemps et dont le début de la lactation est assuré à l'herbe, et de la finition à l'herbe de leurs agneaux. Le niveau d'autonomie fourragère, qui est déjà élevé pour T ( $62 \%)$ atteint $72 \%$ pour E1 et $77 \%$ pour E2.

Les brebis en plein air intégral disposent entre le 15 décembre et fin février d'un foin de bonne qualité à volonté. Le temps de pâturage et les quantités de foin consommées varient fortement d'un jour à l'autre, sous l'effet des conditions météorologiques : de 1 à $7 \mathrm{~h}$ de pâturage par jour par temps de pluie ou par beau temps, et de 0,25 à $1,4 \mathrm{~kg}$ de MS de foin par brebis et par jour. Globalement, sur l'ensemble de la période considérée, on estime que le pâturage couvre la moitié du besoin total des animaux, soit 5 t de MS qui représentent environ $25 \%$ du besoin annuel total en fourrages conservés. Le poids vif et l'état corporel des brebis se maintiennent pendant toute cette période.

\subsection{Constitution des stocks}

Pour les systèmes à faible chargement, la constitution des stocks récoltés en juin et juillet sur des surfaces à faible rendement du fait des apports réduits d'azote est fortement sensible aux aléas climatiques. Cela n'affecte guère la constitution des réserves en E2 en raison du rapport excédentaire entre offre et besoin. En revanche, en E1, la priorité étant donnée au pâturage, le déficit des années sèches se répercute totalement sur les récoltes. La constitution d'un stock de report important $(30 \%$ des besoins annuels du troupeau en E1 et en E2, et $15 \%$ seulement en T) a permis d'apporter une plus grande sécurité des réserves. Constitué les années favorables, ce supplément contribue à maîtriser les excédents des parcelles fauchables.

\subsection{Taux d'utilisation des ressources et évolution de la végétation}

Comparé à $\mathrm{T}$, la disponibilité fourragère de E1 (estimée à $+20 \%$ ) a moins augmenté que sa surface $(+40 \%)$, les rendements par hectare diminuant comme les apports d'azote. Cette évolution de la production, combinée à un accroissement du pâturage, se traduit par une égalisation des taux d'utilisation des ressources entre $\mathrm{E} 1$ et $\mathrm{T}(61 \%$ et $62 \%$, tableau I). 
Tableau I. Données sur les performances zootechniques et les ressources fourragères des exploitations selon le chargement.

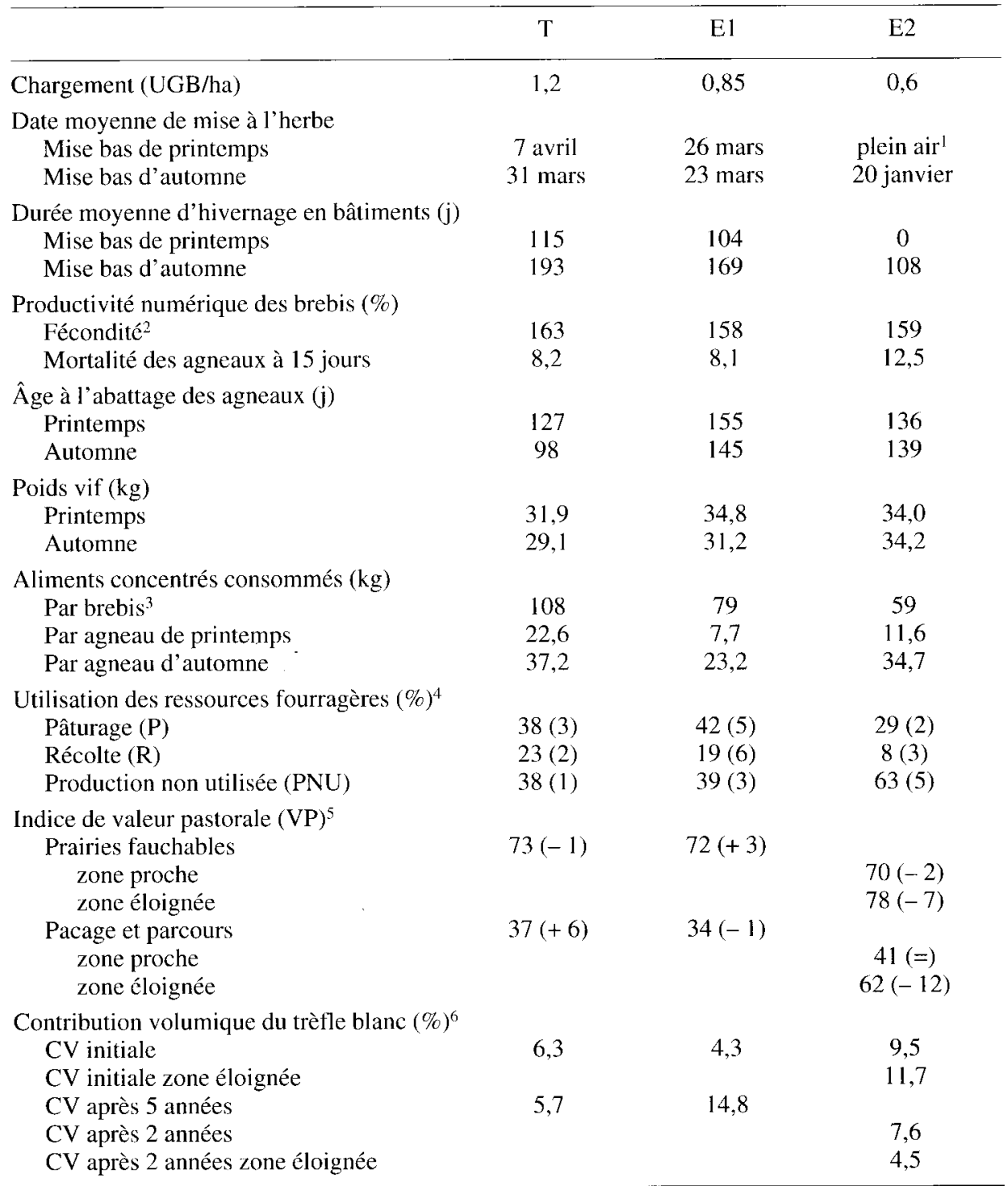

${ }^{1}$ Plein air intégral avec pâturage hivernal et apport de foin ; ${ }^{2}$ Agneaux nés pour 100 femelles mises à la lutte, primipares incluses ; ${ }^{3}$ Consommation du couple mère / agneaux par brebis pouvant mettre bas ; ${ }^{4}$ Production fourragère totale d'un système (PFT) = somme des productions parcellaires estimées par quatre coupes annuelles de repousses sous cages de mise en défens (deux par parcelle) ; les récoltes $(R)$ correspondent aux fourrages fauchés; les quantités d'herbe pâturées $(P)=$ le produit des jours de pâturage par une quantité quotidienne ingérée estimée à $3,1,7,1,5$ et $1 \mathrm{~kg}$ MS pour respectivement les brebis allaitantes, les taries ou gestantes, les agnelles et les agneaux sevrés (quantités réduites de moitié pour les journées de pâturage d'hiver (janvier et février) pour les brebis taries de $\mathrm{E} 2) ; \mathrm{PNU}=\mathrm{PFT}-\mathrm{P}-\mathrm{R}$; entre parenthèses : écart type. ${ }^{5} \mathrm{VP}$ après 5 années de conduite pour T et E1, 2 années pour E2 ; entre parenthèses : variation relative depuis l'état initial en \% ; ${ }^{6}$ Évolution dans les prairies permanentes hors parcours. 
En E2, ce taux d'utilisation est estimé à moins de $40 \%$ et traduit l'importance du déséquilibre entre l'offre et les besoins.

L'évaluation de l'entretien du territoire est faite par le suivi de l'indice de valeur pastorale (VP) des prairies [6] et du recouvrement par les espèces ligneuses, la stabilité de ces critères étant recherchée. L'indice de VP est resté stable dans tous les types de prairies de $\mathrm{T}$ et de $\mathrm{E} 1$, avec pour ce dernier une progression de dix points du taux de trèfle blanc dans les prairies permanentes, hors parcours (tableau I). Cette modification de composition botanique devrait permettre de conserver à terme la qualité et le renouvellement des ressources herbagères.

En E2, la stabilité de la VP n'a été maintenue que dans la zone proche. On observe, en deux ans seulement, une baisse de $7 \%$ sur les zones fauchables et de $12 \%$ sur les prairies non fauchables de la zone éloignée, et une chute de six points du taux de trèfle blanc (tableau I). Le recouvrement des ligneux est passé dans le même temps de 5,2 à $5,8 \%$ sur les parcelles non fauchables de E2.

\section{DISCUSSION ET CONCLUSION}

Les résultats que nous avons obtenus au cours de ces deux essais montrent que les performances zootechniques ont été maintenues à un niveau élevé malgré des adaptations parfois importantes de la conduite des troupeaux et des prairies. Toutefois, l'objectif d'entretien des surfaces risque d'être plus difficile à atteindre, avec seulement 0,6 UGB $\cdot \mathrm{ha}^{-1}$. L'agrandissement des surfaces, avec une forte réduction du chargement, peut cependant être considéré comme une opportunité, et être mis à profit pour réduire l'ensemble des charges d'alimentation du troupeau, et renforcer l'autonomie fourragère.

Pour intégrer les nouvelles surfaces, la nature et l'importance des adaptations à apporter aux conduites dépendent du niveau de chargement. À $0,85 \mathrm{UGB} \cdot \mathrm{ha}^{-1}$, l'effort principal a porté sur la gestion des surfaces fourragères : apports azotés réduits mais ciblés pour permettre l'extension du trèfle blanc, fauches à faibles rendements, conduite permettant d'augmenter le pâturage avec la sortie précoce des brebis, rotation rapide des lots d'animaux sur les parcelles. Les modifications de conduite du troupeau restent mineures, (calendrier de reproduction et élevage des agneaux de printemps), mais elles permettent toutefois d'exercer une pression d'exploitation suffisante des ressources pour assurer de manière pérenne une production d'herbe de bonne qualité répondant aux besoins d'un troupeau productif. Des mesures complémentaires telles que la fauche précoce d'excédents, pratiquée dans les systèmes extensifs [4], ont par ailleurs renforcé l'action du pâturage. Nos résultats sont en accord avec d'autres obtenus dans des milieux pédoclimatiques différents $[3,8]$, montrant qu'une extensification modérée permet la conservation de la qualité des ressources et du niveau des performances animales.

En revanche, à $0,6 \mathrm{UGB} \cdot \mathrm{ha}^{-1}$, la conduite des troupeaux a dû être profondément modifiée. En effet, à ce niveau de chargement, l'excédent de production fourragère par rapport à la capacité de prélèvement du troupeau exige d'allonger fortement la période de pâturage, pour réduire l'accumulation de végétation sur pied préjudiciable à la qualité de la prairie. Le plein air intégral et le pâturage hivernal permettent alors de mobiliser les troupeaux au service de l'entretien des surfaces, mais exigent d'importantes modifications du système d'élevage, avec des mises bas de printemps tardives, ainsi que la surveillance et l'affouragement de troupeaux à l'extérieur en hiver. En outre, la forte infestation parasitaire des surfaces exploitées en hiver doit être prise en compte lors de la saison de pâturage suivante, afin d'éliminer les risques de contamination de catégories d'animaux sensibles. En définitive, ces mesures ne parviennent pas à éviter la diminution des VP sur l'ensemble de la surface 
ni l'extension des buissons. Toutefois, le soin particulier apporté au pâturage des ligneux par des visites fréquentes du troupeau dès le printemps a permis de réduire leur progression à $30 \%$ de celle observée sur des parcelles comparables moins souvent pâturées où le recouvrement est passé de 6,1 à $8,0 \%$. De plus, la baisse de qualité des zones éloignées a été moins forte que celle constatée en 2 ans sur des parcelles homologues moins pâturées, où la VP a chuté de $38 \%$ sur les zones fauchables et $26 \%$ sur les pacages et où le trèfle, très sensible à la sous-exploitation [5] a presque totalement disparu.

En situation de faible chargement, la stratégie a été de répartir au mieux, chaque année, les animaux sur tout le territoire, la consommation et le piétinement contribuant à l'entretien de la végétation. Par ailleurs, l'offre de ressources de qualité aux animaux exigeants, même si elle ne représente qu'une part limitée du total de l'herbe produite, reste un élément clef de la réussite de la conduite extensifiée.

Le maintien d'une productivité animale élevée, avec une bonne autonomie fourragère et l'entretien de la surface fourragère, au moins jusqu'à $0,8 \mathrm{UGB} \cdot$ ha $^{-1}$ ont été obtenus avec des brebis allaitantes, certainement très bien adaptées aux exigences et aux contraintes des systèmes extensifs. L'espèce ovine présente les caractéristiques indispensables à la réussite de tels systèmes : un cycle de production court, offrant une grande souplesse de gestion pour disposer d'animaux à des niveaux de besoins très contrastés ; une importante capacité de tri au pâturage [9] avec la possibilité de valoriser des couverts végétaux de qualité médiocre en pâturage hivernal ; l'aptitude à consommer des ligneux pour contrôler le développement d'espèces indésirables ; un gabarit réduit évitant la dégradation des sols en plein air intégral.

Grâce à ces différentes qualités, et malgré une forte sensibilité au parasitisme et à la prédation, la production ovine allaitante est appelée à jouer un rôle essentiel dans le développement de systèmes de production innovants, très autonomes et soumis par ailleurs à des objectifs contraignants d'entretien des surfaces.

\section{RÉFÉRENCES}

[1] Benoît M., Laignel G., Liénard G., Orientations des exploitations ovines en Massif Central Nord. Observations auprès d'un échantillon d'exploitations de l'Auvergne et du Limousin, LEE-Inra, 1992, $18 \mathrm{p}$.

12] Benoît M., Laignel G., Liénard G., Exploitations ovines en Massif Central Nord. Quels résultats en 1991 ? LEE-Inra, 1993, 27 p.

[3] Chabosseau J.M., Extensive management of grasslands in Central France : Effects on animal performances and economical efficiency. Proc. XVIII Int. Grassl Cong., Canada, session $29,1997,33$ p.

[4] Chabosseau J.M., Dedieu B., La prairie temporaire et les interventions mécaniques en système ovin extensif : exemples en Montmorillonnais, Fourrages 151 (1997) 351-372.

[5] Leconte D., Luxen P., Bourcier J.F., Raisonner l'entretien des prairies et le choix des techniques de rénovation, Fourrages 153 (1998) 15-29.

[6] Loiseau P., de Montard F.X., Gachon L., Aspects biologiques et techniques de la remise en exploitation des hauts pâturages degradés des Monts Dore, in : Utilisation par les ruminants des pâturages d'altitude et parcours méditerranéens, Inra publications, Versailles 1979 , pp. 57-133.

[7] Louault F., de Montard F.X., Brelurut A., Thériez M., Pailleux J.Y., Benoît M., Liénard G., Extensification en élevage ovin par agrandissement des surfaces. Adaptation de la gestion des prairies, Fourrages 154 (1998) 217-237.

[8] Marriott C., Gordon I., Extensification of sheep grazing systems: effects on soil nutrients, species composition and animal production. XVIII Int. Grassl. Cong., 8-19 June 1997, Canada, Session 15 , pp. $3-4$.

[9] Prache S., Roguet C., Petit M., How degree of selectivity modifies foraging behaviour of dry ewes on reproductive compared to vegetative sward structure, Appl. Anim. Behav. Sci. 57 (1998) 91-108.

[10] Thériez M., Brelurut A., Pailleux J.Y., Benô̂t M., Liénard G., Louault F., de Montard F.X., Extensification en élevage ovin viande par agrandissement des surfaces fourragères. Résultats zootechniques et économiques de 5 ans d'expérience dans le Massif Central nord, Prod. Anim. 10 (2) (1997) 14 l-152. 